$\begin{array}{cl}\text { PRAMANA } & \text { (C) Indian Academy of Sciences } \\ \begin{array}{c}\text { journal of } \\ \text { physics }\end{array} & \begin{array}{l}\text { Vol. 69, No. } 5 \\ \text { November } 2007 \\ \text { pp. } 915-919\end{array}\end{array}$

\title{
Lepton distribution in top decay: A probe of new physics and top-polarization
}

\author{
ROHINI M GODBOLE ${ }^{1, *}$, SAURABH D RINDANI ${ }^{2}$ and RITESH K SINGH 3 \\ ${ }^{1}$ Center for High Energy Physics, Indian Institute of Science, Bangalore 560 012, India \\ ${ }^{2}$ Physical Research Laboratory, Ahmedabad 380 009, India \\ ${ }^{3}$ Laboratoire de Physique Théoretique, 91405 Orsay Cedex, France \\ ${ }^{*}$ E-mail: godbolerm@gmail.com
}

\begin{abstract}
We investigate the possibilities of studying new physics in various processes of $t$-quark production using kinematical distributions of the secondary lepton coming from decay of $t$ quarks. We show that the angular distributions of the secondary lepton are insensitive to the anomalous $t b W$ vertex and hence are pure probes of new physics in a generic process of $t$-quark production. The effects of $t$ polarization on the distributions of the decay lepton are demonstrated for top-pair production process at a $\gamma \gamma$-collider mediated by a heavy Higgs boson.
\end{abstract}

Keywords. Top; polarization; anomalous top coupling.

PACS Nos 14.65.Ha; 13.88.+e; 13.85.Qk

\section{Introduction}

As a result of the large mass of top quark and the consequent large coupling to the longitudinal component of the $W$ boson, it decays prior to hadronization. Hence, the spin of the decaying top quark leaves its imprint on the kinematical distributions of the decay products: the $W$ boson and the $b$ quark. Possible new physics of electro-weak symmetry breaking (EWSB) may alter $t$ quark coupling with the $W$ boson leading to changed decay width and distributions. Further, new physics may also appear in its production process potentially affecting kinematical distributions and possibly polarization. The simultaneous presence of new physics both in production and decay processes of top quark may complicate the analysis and it may become difficult to probe new physics couplings of top quark. However, if one can construct observables that are sensitive to production and decay mechanism independent of each other, the analysis can be greatly simplified. It has been shown that the angular distribution of the leptons from the decay of top quark is insensitive to the anomalous $t b W$ coupling in the $2 \rightarrow 2$ processes of top quark production [1] and also for a general process [2]. Here we extend the result by relaxing the approximations used in the earlier analysis, for a general $2 \rightarrow n$ process [3]. 


\section{Anomalous $t b W$ interaction and lepton distribution}

We treat the $t b W$ vertex in a model independent way by parameterizing it as

$$
\Gamma^{\mu}=\frac{-i g}{\sqrt{2}}\left[\gamma^{\mu}\left(f_{1 L} P_{L}+f_{1 R} P_{R}\right)-\frac{i \sigma^{\mu \nu}}{m_{W}}\left(p_{t}-p_{b}\right)_{\nu}\left(f_{2 L} P_{L}+f_{2 R} P_{R}\right)\right]
$$

For the SM we have $f_{1 L}=1$ and the anomalous couplings $f_{1 R}=f_{2 L}=f_{2 R}=0$. Further we note that the contribution from $f_{1 R}$ and $f_{2 L}$ is proportional to $m_{b}$ and will be absent in the vanishing $m_{b}$ limit. To show the decoupling of lepton angular distribution from anomalous top coupling we assume

1. $t$-quark is on-shell, i.e. using narrow-width approximation for top quark and

2. anomalous couplings $f_{1 R}, f_{2 R}$ and $f_{2 L}$ are small, we retain terms linear in them.

Using narrow-width approximation for top quark we can write the matrix element square as

$$
|M|^{2}=\frac{\pi \delta\left(p_{t}^{2}-m_{t}^{2}\right)}{m_{t} \Gamma_{t}} \sum_{\lambda, \lambda^{\prime}} \rho\left(\lambda, \lambda^{\prime}\right) \Gamma\left(\lambda, \lambda^{\prime}\right) ; \quad \begin{aligned}
\rho\left(\lambda, \lambda^{\prime}\right) & =M_{\rho}(\lambda) M_{\rho}^{*}\left(\lambda^{\prime}\right) \\
\Gamma\left(\lambda, \lambda^{\prime}\right) & =M_{\Gamma}(\lambda) M_{\Gamma}^{*}\left(\lambda^{\prime}\right) .
\end{aligned}
$$

With this the differential cross-section for $2 \rightarrow n$ process of top production followed by its decay can be written as

$$
\begin{aligned}
\mathrm{d} \sigma= & \sum_{\lambda, \lambda^{\prime}}\left[\frac{(2 \pi)^{4}}{2 I} \rho\left(\lambda, \lambda^{\prime}\right) \delta^{4}\left(k_{A}+k_{B}-p_{t}-\sum_{i}^{n-1} p_{i}\right) \frac{\mathrm{d}^{3} p_{t}}{2 E_{t}(2 \pi)^{3}} \prod_{i}^{n-1} \frac{\mathrm{d}^{3} p_{i}}{2 E_{i}(2 \pi)^{3}}\right] \\
& \times\left[\frac{1}{\Gamma_{t}}\left(\frac{(2 \pi)^{4}}{2 m_{t}} \Gamma\left(\lambda, \lambda^{\prime}\right) \delta^{4}\left(p_{t}-p_{b}-p_{\nu}-p_{\ell}\right) \frac{\mathrm{d}^{3} p_{b}}{2 E_{b}(2 \pi)^{3}} \frac{\mathrm{d}^{3} p_{\nu}}{2 E_{\nu}(2 \pi)^{3}}\right) \frac{\mathrm{d}^{3} p_{\ell}}{2 E_{\ell}(2 \pi)^{3}}\right] .
\end{aligned}
$$

The first term in eq. (3) can be written as $\mathrm{d} \sigma_{2 \rightarrow n}\left(\lambda, \lambda^{\prime}\right) \mathrm{d} E_{t} \mathrm{~d} \cos \theta_{t}$ after integration in the lab frame, while we integrate the second term in the rest frame of the decaying top quark as

$$
\begin{aligned}
\frac{1}{\Gamma_{t}} & \frac{(2 \pi)^{4}}{2 m_{t}} \int \frac{\mathrm{d}^{3} p_{\ell}}{2 E_{\ell}(2 \pi)^{3}} \frac{\mathrm{d}^{3} p_{b}}{2 E_{b}(2 \pi)^{3}} \frac{\mathrm{d}^{3} p_{\nu}}{2 E_{\nu}(2 \pi)^{3}} \\
& \times \Gamma\left(\lambda, \lambda^{\prime}\right) \delta^{4}\left(p_{t}-p_{b}-p_{\nu}-p_{\ell}\right) \\
= & \frac{1}{32 \Gamma_{t} m_{t}} \frac{E_{\ell}}{(2 \pi)^{4}} \frac{\left\langle\Gamma\left(\lambda, \lambda^{\prime}\right)\right\rangle}{m_{t} E_{\ell}} \mathrm{d} E_{\ell} \mathrm{d} \Omega_{\ell} \mathrm{d} p_{W}^{2} .
\end{aligned}
$$

Here the angular brackets indicate averaging over azimuthal angle between $b$ quark and decay lepton. In the rest frame of the top quark we have 


$$
\left\langle\Gamma\left(\lambda, \lambda^{\prime}\right)\right\rangle=g^{4} m_{t} E_{\ell}^{0}\left|\Delta_{W}\left(p_{W}^{2}\right)\right|^{2} A\left(\lambda, \lambda^{\prime}\right) \times F\left(E_{\ell}^{0}\right),
$$

where $\Delta_{W}\left(p_{W}^{2}\right)=\frac{1}{p_{W}^{2}-m_{W}^{2}+i \Gamma_{W} m_{W}}$ is the $W$-propagator, $A( \pm, \pm)=\left(1 \pm \cos \theta_{\ell}\right)$, $A( \pm, \mp)=\sin \theta_{\ell} e^{ \pm \phi_{\ell}}$ is the pure angular factor and $F\left(E_{\ell}^{0}\right)$ is given as

$$
\begin{aligned}
F\left(E_{\ell}^{0}\right)= & {\left[\left(m_{t}^{2}-m_{b}^{2}-2 p_{t} \cdot p_{l}\right)\left(\left|f_{1 L}\right|^{2}+\Re\left(f_{1 L} f_{2 R}^{*}\right) \frac{m_{t}}{m_{W}} \frac{p_{W}^{2}}{p_{t} \cdot p_{l}}\right)\right.} \\
& \left.-2 \Re\left(f_{1 L} f_{2 L}^{*}\right) \frac{m_{b}}{m_{W}} p_{W}^{2}-\Re\left(f_{1 L} f_{1 R}^{*}\right) \frac{m_{b} m_{t}}{p_{t} \cdot p_{l}} p_{W}^{2}\right]
\end{aligned}
$$

is angle independent factor that depends upon the lepton energy. It should be noted here that all the anomalous couplings appear only in $F\left(E_{\ell}^{0}\right)$. Combining the production and decay part, we have

$$
\begin{aligned}
\mathrm{d} \sigma= & \frac{1}{32 \Gamma_{t} m_{t}(2 \pi)^{4}}\left[\sum_{\lambda, \lambda^{\prime}} \mathrm{d} \sigma_{2 \rightarrow n}\left(\lambda, \lambda^{\prime}\right) \times g^{4} A^{\mathrm{c} . \mathrm{m} .}\left(\lambda, \lambda^{\prime}\right)\right] \\
& \times \mathrm{d} E_{t} \mathrm{~d} \cos \theta_{t} \mathrm{~d} \cos \theta_{\ell} \mathrm{d} \phi_{\ell} \times E_{\ell} F\left(E_{\ell}\right) \mathrm{d} E_{\ell} \mathrm{d} p_{W}^{2} .
\end{aligned}
$$

Further, we have $\Gamma_{t} \propto \int E_{\ell} F\left(E_{\ell}\right) \mathrm{d} E_{\ell} \mathrm{d} p_{W}^{2}$. Thus, if we integrate over $E_{\ell}$ in eq. (7), the factor containing anomalous couplings cancels between the numerator and $\Gamma_{t}$ in the denominator. This proves that the differential rates are independent of anomalous $t b W$ couplings once we integrate over lepton energy. Hence, the angular distribution of lepton is independent of anomalous $t b W$ couplings in any inertial frame of reference and thus sensitive only to the production mechanism and possible new physics therein. On the other hand, the energy distribution of the decay lepton in the lab frame depends on the $t b W$ vertex, i.e. possible new physics in top decay, and also on energy-angular distribution of the produced top quarks, i.e. possible new physics in top production. However, the $E_{\ell}^{0}$ distribution in the rest frame of the decaying top quark is given by $\mathrm{d} \sigma / \mathrm{d} E_{\ell}^{0} \propto \int E_{l}^{0} F\left(E_{l}^{0}\right) \mathrm{d} p_{W}^{2}$, hence sensitive only to the decay vertex with proportionality constant absorbing the information about production mechanism. In other words, the $E_{\ell}^{0}$ distribution (in the rest frame of top quark) provides sensitivity to the anomalous $t b W$ coupling independent of the production mechanism of top quark. To summarize, the decay lepton distribution can provide separate and pure probes of possible new physics in production and decay of top quarks.

\section{Polarization of top quark}

Denoting the first line of eq. (3) by $\sigma\left(\lambda, \lambda^{\prime}\right)$, one can write the polarization density matrix of the top quark as

$$
P_{t}=\frac{1}{2}\left(\begin{array}{cc}
1+\eta_{3} & \eta_{1}-i \eta_{2} \\
\eta_{1}+i \eta_{2} & 1-\eta_{3}
\end{array}\right), \quad \text { where } \begin{aligned}
& \eta_{3}=(\sigma(+,+)-\sigma(-,-)) / \sigma_{\text {tot }}, \\
& \eta_{1}=(\sigma(+,-)+\sigma(-,+)) / \sigma_{\text {tot }}, \\
& i \eta_{2}=(\sigma(+,-)-\sigma(-,+)) / \sigma_{\text {tot }}
\end{aligned}
$$


Rohini M Godbole, Saurabh D Rindani and Ritesh K Singh

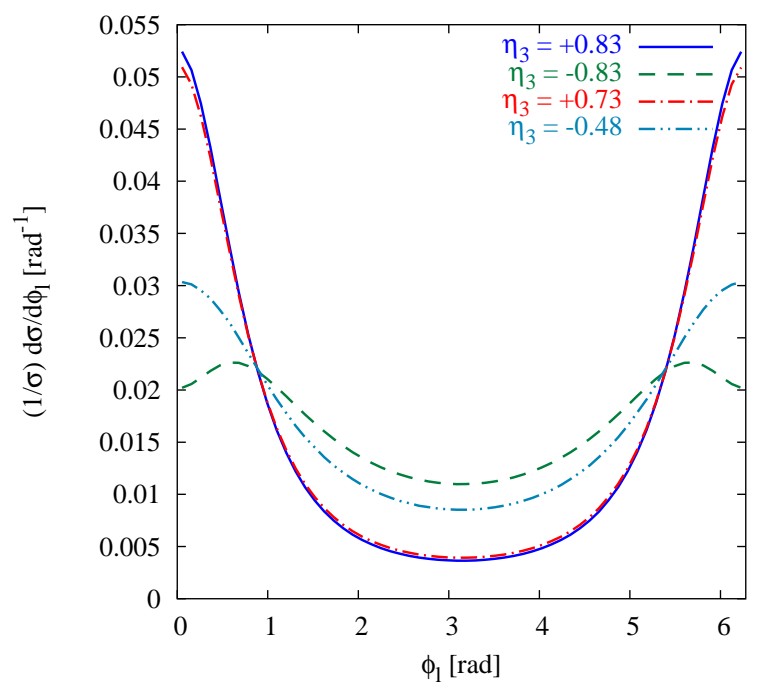

Figure 1. The distribution in the azimuthal angle of the lepton in the lab frame for different top polarizations.

and $\sigma_{\text {tot }}=\sigma(+,+)+\sigma(-,-)$. Here $\eta_{3}$ is the longitudinal polarization of the top quark or the average helicity, $\eta_{2}$ is the transverse polarization normal to the production plane and $\eta_{1}$ is the transverse polarization in the production plane. These polarizations can be calculated from the angular distribution of leptons as

$$
\frac{\eta_{i}}{2}=\frac{\sigma\left(p_{\ell} \cdot s_{i}<0\right)-\sigma\left(p_{\ell} \cdot s_{i}>0\right)}{\sigma\left(p_{\ell} \cdot s_{i}<0\right)+\sigma\left(p_{\ell} \cdot s_{i}>0\right)}, \text { for } i=1,2,3
$$

where $s_{i} \cdot s_{j}=-\delta_{i j}$ and $p_{t} \cdot s_{i}=0$. For $p_{t}^{\mu}=E_{t}\left(1, \beta_{t} \sin \theta_{t}, 0, \beta_{t} \cos \theta_{t}\right)$, we have $s_{1}^{\mu}=\left(0,-\cos \theta_{t}, 0, \sin \theta_{t}\right), s_{2}^{\mu}=(0,0,1,0)$ and $s_{3}^{\mu}=E_{t}\left(\beta_{t}, \sin \theta_{t}, 0, \cos \theta_{t}\right) / m_{t}$. The measurement of $\eta_{i}$ requires knowledge of $s_{i} \mathrm{~s}$. In other words, one requires to fully or partially reconstruct top momentum in the lab frame. Alternatively, one can look at the azimuthal distribution of leptons with respect to the top production plane as (at least) a qualitative probe of top polarization. For demonstration, we choose $\gamma \gamma \rightarrow t \bar{t}$ with and without Higgs mediation and polarized photon beams. With the help of variation in the photon polarization and the presence and absence of Higgs exchange we generate ensemble of top quarks with varying polarization and show the decay lepton azimuthal distribution in figure 1 . We note that for this example we have $\eta_{1}=\eta_{2}=0$ and the values of $\eta_{3}$ are indicated on the plot. We see that the distribution is peaked at the top production plane $\left(\phi_{\ell}=0,2 \pi\right)$ for positively polarized top and the height of peak decreases as polarization changes from positive to negative value. For large negative value of polarization $(-0.83)$ there is a dip in the distribution in place of the peak near the top production plane. This qualitative feature of the azimuthal distribution can be converted into quantitative measure after establishing the correlation between $\eta_{3}$ and height of the peak for a given process. 


\section{Lepton distribution in top decay}

To conclude, we show that the lepton angular distribution is sensitive to top polarization and production mechanism independent of anomalous $t b W$ couplings and $E_{\ell}^{0}$ distribution is sensitive to anomalous $t b W$ coupling independent of top production mechanism. Further, we show that azimuthal distribution of decay lepton in the lab frame can be used to probe polarization of decaying top quark.

\section{References}

[1] S D Rindani, Pramana - J. Phys. 54, 791 (2000)

B Grzadkowski and Z Hioki, Phys. Lett. B476, 87 (2000)

Z Hioki, hep-ph/0104105

K Ohkuma, Nucl. Phys. (Proc. Suppl.) B111, 285 (2002)

R M Godbole, S D Rindani and R K Singh, Phys. Rev. D67, 095009 (2003), Erratum, Phys. Rev. D71, 039902 (2005)

B Grzadkowski and Z Hioki, Phys. Lett. B529, 82 (2002)

[2] B Grzadkowski and Z Hioki, Phys. Lett. B557, 55 (2003)

Z Hioki, hep-ph/0210224

[3] R M Godbole, S D Rindani and R K Singh, arXiv:hep-ph/0605100 